\title{
Limited clinical value of exhaled volatile organic compound measurements in childhood asthma
}

\section{To the Editor:}

Noninvasive, clinically applicable measurements of biomarkers for diagnosis of asthma in early life are needed. We hypothesised that profiles of volatile organic compounds (VOCs) in exhaled breath samples may serve such a purpose.

Chronic airway inflammation involves processes such as oxidative stress and, thereby, release of VOCs that can be detected in breath samples [1]. Promising VOC results for differentiation between adults with and without asthma have been published previously [2,3]. In children, we have demonstrated effective differentiation between healthy and asthmatic subjects using gas chromatography-mass spectrometry (GC-MS) [1], and between preschoolers with and without acute wheezing using GC-MS [4] and electronic nose (eNose) technology [5]. However, there is a lack of studies exploring the utility of VOC profiling in a paediatric outpatient clinical setting.

In this study, we took two approaches within modern breathomics, the eNose and GC-MS techniques, to explore the potential of VOC profiles in an outpatient clinic to discriminate between children with and without asthma.

We recruited children from a single tertiary paediatric asthma outpatient clinic with a diagnosis of asthma who were diagnosed based on a typical symptom history (e.g. exercise-induced symptoms, prolonged nocturnal cough, recurrent cough outside common cold or symptoms causing wakening at night) in conjunction with lung function deficits such as variable airflow obstruction, bronchial reversibility and bronchial hyperresponsiveness. We excluded children with an asthma exacerbation or airway infection within the previous 2 weeks and children treated with oral corticosteroids within the previous month. The only restriction prior to breath collection was withholding any asthma medication on the day of sampling. Atopy among the asthma cases was defined as any specific IgE level $>0.35 \mathrm{kU} \cdot \mathrm{L}^{-1}$ and/or any positive skin prick test result.

Age-matched children without previous or current asthma, allergy or eczema were recruited from the local community and used as healthy controls. The parents were interviewed about their child's history of asthma, allergy or eczema using the International Study of Asthma and Allergies in Childhood core questions [6]. Lung function and allergic sensitisation status was not measured among the healthy controls.

Approvals from the Danish National Committee on Health Research Ethics and the Danish Data Protection Agency, and parental oral and written informed consent were obtained prior to any study related procedures.

We aimed to collect breath samples by three different methods at the same visit for all children. 1) Collection of exhaled air in a $750-\mathrm{mL}$ bag made of chemically inert material (GaSampler System; Quintron Instrument Company, Milwaukee, WI, USA) by tidal breathing, followed by analysis with Cyrano 320 eNose (Smiths Detection Inc., Pasadena, CA, USA). Part of the breath sample was trapped in a carbon tube and shipped for later analysis by GC-MS as previous detailed [5]. 2) As in method 1 but

@ERSpublications

Exhaled volatile organic compound measurements do not aid the clinician diagnosing asthma in children http://ow.ly/Z2d930lpZ60

Cite this article as: Fuglsbjerg MG, Rasmussen MA, Hansen KS, et al. Limited clinical value of exhaled volatile organic compound measurements in childhood asthma. ERJ Open Res 2018; 4: 00026-2018 [https://doi.org/10.1183/23120541.00026-2018]. 
with a VOC filter (A1; North Safety, Middelburg, the Netherlands) attached to the inspiratory port of the valve. 3) Collection of exhaled air in a modified metallic spacer (Nebuhaler) by tidal breathing with simultaneous eNose analysis, using a VOC filter (A1) attached to the inspiratory port of the valve.

All three containers were connected to a single shutter patient valve (Ambu Silicone Resuscitator; Ambu A/S, Ballerup, Denmark) and facial mask (Ambu UltraSeal Paediatric) covering the mouth and nose.

The eNose data were preprocessed using second derivative by the Savitzky-Golay method and scaled to unit variance to correct for a common offset across all variables within samples. The GC-MS data were preprocessed as in our earlier studies [5] and filtered by removing variables with <7\% positive registrations, reducing the number of identified metabolites from 2065 to 1168 ; these were subsequently square root transformed to account for distributional skewness and scaled to unit variance prior to modelling.

We subsequently applied a range of statistical analyses to try to differentiate between children with asthma and healthy controls in order to comprehensively analyse our data for such information. This included using: unsupervised models, i.e. models not trained on the outcome of interest (asthma), based on dimensionality reduction by principal component analysis (PCA) [7]; and supervised models, i.e. models trained on the outcome of interest (asthma) for classification including 1) linear discriminate analysis (LDA) [8], 2) least absolute shrinkage and selection operator (LASSO) with embedded variable selection and permutation testing for the GC-MS analysis and leave-one-out cross-validation [9], and 3) nonlinear random forest models [10].

As a secondary exploratory analysis, we investigated whether lung function, bronchial reversibility and atopy status differed among children classified as asthmatics by their VOC profiles. This was done using the extracted posterior probabilities for asthma from the LDA models.

$\mathrm{R}$ version 3.3.3 with the packages MASS, glmnet, and pROC for LDA, logistic regression with LASSO variable selection and receiver operating characteristic curves were used for the data analysis.

A total of 55 children aged 2-14 years participated in the study: 31 asthmatics (20 (64.5\%) male, mean \pm SD age $8.8 \pm 2.9$ years) and 24 age-matched healthy controls (13 (54.2\%) male and age $9.4 \pm 3.6$ years). $65 \%$ of the asthmatic children were regarded as atopic based on symptoms of allergy and a positive skin prick test and/or elevated specific IgE level. The asthmatic children had a forced expiratory volume in $1 \mathrm{~s}$ (FEV1) of $90.1 \pm 12.4 \%$ predicted and $30(96.8 \%)$ of the children were receiving maintenance treatment with inhaled corticosteroids. There was no statistically significant difference in age or sex comparing the group of asthmatic children with the group of healthy children.

All 55 (100\%) children managed to fill both bags for methods 1 and 2, and 54 (98.2\%) children completed method 3 with the spacer. Tubes for GC-MS analysis were obtained from 50 (90.9\%) children.

The unsupervised PCA models did not reveal any associations but when using supervised models, the analyses of the VOC profiles indicated a possible discrimination between asthmatic and healthy children
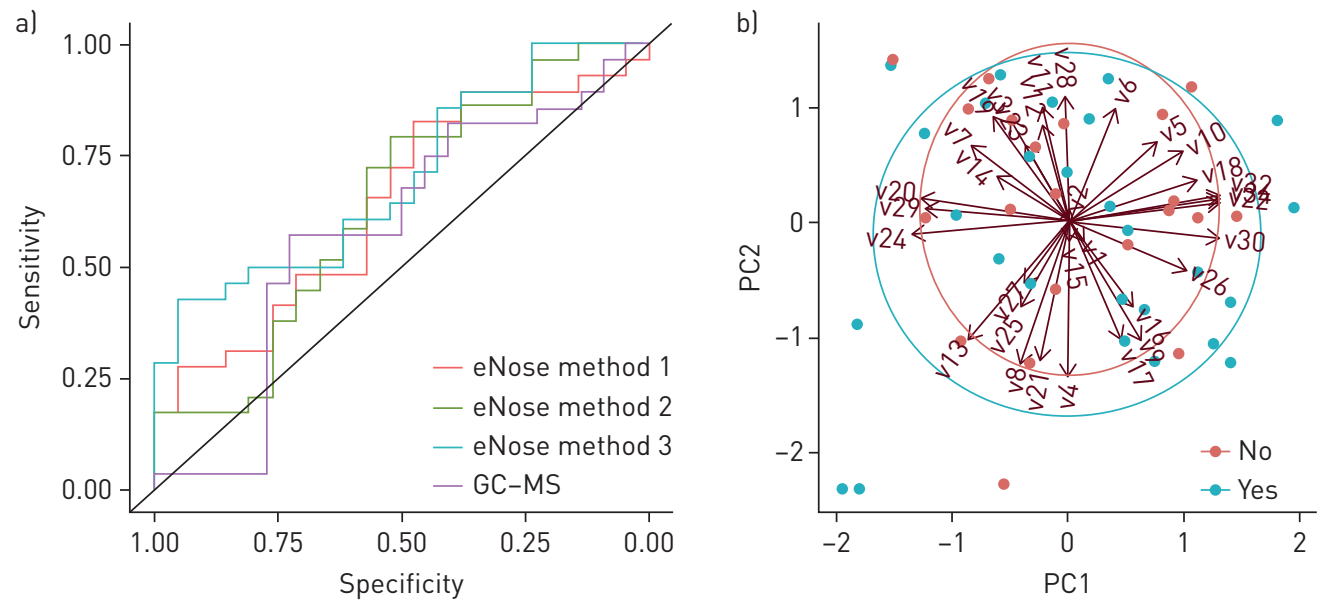

FIGURE 1 a) Receiver operator characteristic curves illustrating the area under the curve for the three electronic nose (eNose) methods and for the gas chromatography-mass spectrometry (GC-MS) profiles to discriminate between children with asthma and healthy controls. b) Driving principal components (PCs) for eNose method 3 including class membership (asthma, yes or no). 
based on eNose data, with the strongest signal using sampling method 3 ( $p=0.008$ ) followed by method 1 and 2 (both $\mathrm{p}=0.046$ ). However, cross-validation values were weak with a correct classification of a maximum of $70 \%$ for method 3, and $64 \%$ for both methods 1 and 2. Analyses of GC-MS data did not show a significant separation by any method $(\mathrm{p}>0.16)$ with a correct classification of only $58 \%$ (figure 1 ). Results were similar by nonlinear random forest classification (data not shown).

Age had no influence on the individual VOC variables measured by either method. In addition, when stratifying the children based on age tertiles, i.e. age 2-7, 8-10 and 11-14 years, we observed no discriminatory ability of the VOC profiles by either method with respect to asthma status $(\mathrm{p}>0.06)$. However, these post hoc analyses suffer from low power.

FEV1 \% predicted and bronchial reversibility were not correlated with the VOC-determined probability of asthma from the supervised LDA models using any of the three different eNose sampling methods ( $p>0.40$ and $p>0.35$, respectively). In addition, the VOC profiles from method 1 showed a trend of discrimination between asthmatics with versus without atopy $(p=0.07)$, whereas such discrimination was not apparent using methods 1 and $2(\mathrm{p}>0.77)$. Thus, the VOC profiles did not determine a specific asthma phenotype.

We investigated the clinical utility of VOC profiles in breath samples to differentiate between children with and without asthma in a paediatric outpatient setting. Based on our results, we found some discriminatory power of the eNose technology at the population level but not at the individual level.

We specifically aimed to investigate the clinical utility of a simple procedure and therefore used a setup and sampling method with few restrictions. This is in contrast to studies by other research groups [2, 3], and to our own previous studies in which we included limitations regarding exhaled flow range during sampling and sampled larger volumes $[1,11]$. The different approaches may explain why previous studies have shown good discriminatory capacity $[1,3]$. However, overly complicated procedures will be of limited use within paediatrics, especially among preschool children, where the need for improved diagnostic methods is most urgent.

Using GC-MS profiles, we were not able to discriminate between asthmatic and healthy children, which is in contrast to a previous study of 252 children aged 2-6 years showing that GC-MS profiles significantly discriminated between 202 children with recurrent wheeze and 50 healthy controls, with $73 \%$ correct classification [4]. The main differences between that and our study is a lower mean age, withdrawal of inhaled corticosteroids 4 weeks prior to air sampling and a much larger number of cases, which overall, may increase the power to detect differences. However, another GC-MS study of 40 children with asthma aged 6-16 years [11], i.e. similar age and sample size as our study, was able to show discrimination between children with versus without an exacerbation with a correct classification of $91 \%$, making power a less likely factor.

We were able to detect some discriminatory ability of the eNose profiles with a maximum correct classification of $70 \%$. These findings align with another eNose study of 178 young children [5] showing correct classification of recurrent wheezers during exacerbations with area under the curve (AUC) values of 0.77-0.81 and outside exacerbations with AUC values of 0.67-0.84. In addition, eNose studies in adults have shown discrimination between healthy subjects and subjects with asthma [3] and chronic obstructive pulmonary disease [2].

The asthmatic children in our study were all followed at a tertiary paediatric asthma centre and were well controlled for their asthma. Thus, a possible explanation for our negative findings could be a low disease activity resulting in small concentrations of inflammatory derived VOCs in their breath samples. In addition, the sample size of only $750 \mathrm{~mL}$ total breath may have provided VOC concentrations below the limit of detection, especially from the older participants who filled the bags with fewer breaths. Another factor that may have biased the results towards the null is that only one child with asthma was steroid naive and that inhaled corticosteroids were solely withheld on the day of air sampling. It is plausible that VOC profiles are more discriminatory in patients with ongoing airway inflammation, i.e. treatment-naïve patients or patient withholding their controller medication. Finally, it is a limitation of our study that we did not perform lung function tests among the healthy controls to rule out latent asthma.

In conclusion, our study suggests that VOC profiling may have a future role in diagnosis and monitoring of childhood asthma. In particular, the eNose is easy to use and does not require a laboratory setup like that of GC-MS profiling. However, methodological challenges such as an inability to calibrate the eNose and drift of the sensors represent limitations. In addition, there is a need to standardise the air sampling procedure with respect to air volume and withdrawal of maintenance treatment prior to sampling. Thus, further refinement and methodological improvements are needed before breathomics can be introduced in clinical practice. 
Maria G. Fuglsbjerg ${ }^{1}$, Morten A. Rasmussen ${ }^{1,2}$, Kirsten S. Hansen ${ }^{3}$, Agnieszka Smolinska ${ }^{4}$, Jan W. Dallinga ${ }^{4}$, Frederik-Jan van Schooten ${ }^{4}$, Jakob Stokholm ${ }^{1}$, Klaus Bønnelykke ${ }^{1}$, Hans Bisgaard ${ }^{1}$ and Bo L. Chawes ${ }^{1}$

${ }^{1}$ COPSAC, Copenhagen Prospective Studies on Asthma in Childhood, Herlev and Gentofte Hospital, University of Copenhagen, Copenhagen, Denmark. ${ }^{2}$ Dept of Food Science, Faculty of Science, University of Copenhagen, Denmark. ${ }^{3}$ The Pediatric Dept, Herlev and Gentofte Hospital, University of Copenhagen, Copenhagen, Denmark. ${ }^{4}$ Dept of Pharmacology and Toxicology, School of Nutrition and Translational Research in Metabolism (NUTRIM), Maastricht University Medical Centre, Maastricht, The Netherlands.

Correspondence: Hans Bisgaard, COPSAC, Copenhagen Prospective Studies on Asthma in Childhood, Herlev and Gentofte Hospital, University of Copenhagen, Copenhagen, Denmark.

E-mail: bisgaard@copsac.dk

Received: Feb 122018 | Accepted after revision: Aug 012018

Conflict of interest: M.G. Fuglsbjerg has nothing to disclose. M.A. Rasmussen has nothing to disclose. K.S. Hansen has nothing to disclose. A. Smolinska has nothing to disclose. J.W. Dallinga has nothing to disclose. F-J. van Schooten is indicated as the inventor on a pending patent for a method for the diagnosis of airway disease inflammatory subtypes by the Université de Liège, Centre Hospitalier Universitaire de Liège and Universiteit Maastricht. J. Stokholm has nothing to disclose. K. Bønnelykke has nothing to disclose. H. Bisgaard reports receiving grants from the Lundbeck Foundation and the Ministry of Health, Denmark, during the conduct of the study; consultancy fees from Boehringer Ingelheim and Chiesi Pharmaceuticals; and grants from Novo Nordisk foundation, outside the submitted work. B.L. Chawes has nothing to disclose.

\section{References}

1 Smolinska A, Klaassen EMM, Dallinga JW, et al. Profiling of volatile organic compounds in exhaled breath as a strategy to find early predictive signatures of asthma in children. PLoS ONE 2014; 9: e95668.

2 Fens N, Zwinderman AH, van der Schee MP, et al. Exhaled breath profiling enables discrimination of chronic obstructive pulmonary disease and asthma. Am J Respir Crit Care Med 2009; 180: 1076-1082.

3 Dragonieri S, Schot R, Mertens BJA, et al. An electronic nose in the discrimination of patients with asthma and controls. J Allergy Clin Immunol 2007; 120: 856-862.

4 van de Kant KDG, van Berkel JJBN, Jöbsis Q, et al. Exhaled breath profiling in diagnosing wheezy preschool children. Eur Respir J 2013; 41: 183-188.

5 van der Schee MP, Hashimoto S, Schuurman AC, et al. Altered exhaled biomarker profiles in children during and after rhinovirus-induced wheeze. Eur Respir J 2015; 45: 440-448.

6 Asher MI, Keil U, Anderson HR, et al. International Study of Asthma and Allergies in Childhood (ISAAC): rationale and methods. Eur Respir J 1995; 8: 483-491.

7 Bro R, Smilde AK. Principal component analysis. Anal Methods 2014; 6: 2812-2831.

8 Balakrishnama S, Ganapathiraju A. Linear Discriminant Analysis - A Brief Tutorial. Starkville, Institute for Signal and Information Processing, 1998.

9 Friedman J, Hastie T, Tibshirani R. Regularization paths for generalized linear models via coordinate descent. J Stat Soft 2010; 33: https://doi.org/10.18637/jss.v033.i01.

10 Liaw A, Wiener M. Classification and regression by randomForest. $R$ News 2002; 2: 18-22.

11 Robroeks CM, van Berkel JJ, Jöbsis Q, et al. Exhaled volatile organic compounds predict exacerbations of childhood asthma in a 1-year prospective study. Eur Respir J 2013; 42: 98-106. 